APPLICATIONES MATHEMATICAE

25,1 (1998), pp. 101-112

T. NADZIEJA (Zielona Góra)

A. RACZYŃSKI (Wrocław)

\title{
A SINGULAR RADIALLY SYMMETRIC PROBLEM IN ELECTROLYTES THEORY
}

Abstract. Existence of radially symmetric solutions (both stationary and time dependent) for a parabolic-elliptic system describing the evolution of the spatial density of ions in an electrolyte is studied.

1. Introduction. We are interested in the temporal evolution of the spatial density of ions in an electrolyte confined to a container $\Omega$. We consider a simplified, idealized situation when the electrolyte contains only cations, i.e. positively charged particles.

If $u(x, t)$ is the density of ions, $\varphi$ the electric potential produced by the ions through collective effects, and $\widetilde{\varphi}$ a given external electric potential, then the evolution of $u$ is described by the parabolic-elliptic system

$$
\begin{gathered}
u_{t}=\nabla \cdot(\nabla u+u \nabla \Phi), \\
\Delta \varphi=-u, \\
\Phi=\varphi+\widetilde{\varphi} .
\end{gathered}
$$

Here $u, \varphi, \widetilde{\varphi}: \Omega \times \mathbb{R}^{+} \rightarrow \mathbb{R}$, and $\Omega$ is a smooth domain in $\mathbb{R}^{n}$ containing the origin.

For more physical details we refer the reader to [4], [10].

In this paper we assume that $\widetilde{\varphi}$ is of the form $\widetilde{\varphi}(x)=-q^{*} E_{n}(x)$, where $q^{*} \geq 0$ and $E_{n}$ is the fundamental solution of the Laplacian $\left(E_{2}(z)=\right.$ $(2 \pi)^{-1} \log |z|, E_{n}(z)=-\left((n-2) \sigma_{n}\right)^{-1}|z|^{2-n}$ for $n \geq 3, \sigma_{n}$ is the area of the unit sphere in $\left.\mathbb{R}^{n}\right)$. This means that $\widetilde{\varphi}$ is produced by a positive charge $q^{*}$ fixed at the origin.

1991 Mathematics Subject Classification: 35B40, 35K60, 76W05, 82D, 92E.

Key words and phrases: electrodiffusion of ions, nonlinear parabolic equation, radial solutions.

The preparation of this paper was supported by the KBN grant 324/P03/97/07 and NATO grant OUTR.CRG 961130. 
The system (1)-(3) is supplemented with the initial condition

$$
u(x, 0)=u_{0}(x),
$$

and boundary conditions

$$
\begin{gathered}
\varphi=0 \quad \text { on } \partial \Omega, \\
\frac{\partial u}{\partial \nu}+u \frac{\partial \Phi}{\partial \nu}=0 \quad \text { on } \partial \Omega,
\end{gathered}
$$

where $\nu$ denotes the exterior unit normal to $\partial \Omega$. The condition (5) means that the boundary $\partial \Omega$ is grounded, (6) implies that the total charge of ions in $\Omega$ is preserved, i.e. $\int_{\Omega} u(x, t) d x=\int_{\Omega} u_{0}(x) d x=: q_{0}$.

In [1] and [2] it was proved that a local-in-time solution to (1)-(6) with $q^{*}=0$ exists. Moreover, under some assumptions imposed on the dimension $n$ or initial data $u_{0}$, this solution is global and tends to a unique stationary one as $t$ goes to $\infty$.

The problem (1)-(6) with $q^{*}<0$ describes the situation when a charge $q^{*}$ fixed at the origin is surrounded by ions of opposite sign moving in a solute. It is worth noting that the existence of solutions of this problem depends on $n$ and $q^{*}([3])$. For instance, in the radially symmetric case, if $n=2$ and $q^{*}>-4 \pi$ the solution is global, whereas for $q^{*}<-4 \pi$ or $n \geq 3$ and $q^{*}<0$ there are no local-in-time solutions. In our case $q^{*} \geq 0$, the global solution exists for each $q^{*} \geq 0$ and tends asymptotically, as time goes to infinity, to the unique steady state solution.

We assume that $\Omega=\{\|x\| \leq R\}$ is the ball of radius $R$, and we look for radially symmetric solutions of (1)-(6). In this case (1)-(3) reads

$$
\begin{gathered}
u_{t}=r^{1-n}\left(r^{n-1}\left(u_{r}+u \Phi_{r}\right)\right)_{r}, \\
r^{1-n}\left(r^{n-1} \varphi_{r}\right)_{r}=-u, \\
\Phi=\varphi-q^{*} E_{n}(r) .
\end{gathered}
$$

Let $\widetilde{Q}(r, t)$ denote the total charge of moving ions in the ball of radius $r$ at time $t$, i.e.

$$
\widetilde{Q}(r, t)=\sigma_{n} \int_{0}^{r} s^{n-1} u(s, t) d s .
$$

It is easy to check that $Q=q^{*}+\widetilde{Q}$ satisfies the equation

$$
Q_{t}=Q_{r r}-(n-1) r^{-1} Q_{r}-\sigma_{n}^{-1} r^{1-n} Q Q_{r} .
$$

The last equation has the following scaling property: if $Q$ is a solution then $Q(r, t)=\lambda^{2-n} Q\left(\lambda r, \lambda^{2} t\right)$ is also a solution. Hence, without loss of generality, we can consider our problem on the interval $[0,1]$ with boundary and initial data

$$
Q(0, t)=q^{*},
$$




$$
\begin{gathered}
Q(1, t)=q^{*}+q_{0}=: \bar{q}, \\
Q(r, 0)=Q_{0}(r),
\end{gathered}
$$

where $Q_{0}(r)$ is a given, nonnegative, nondecreasing function on $[0,1]$ such that $Q_{0}(0)=q^{*}$ and $Q_{0}(1)=\bar{q}$.

If the equation $(10)$ is considered on $[0, \infty)$ then the only boundary data is $(11)$, and the initial data $Q_{0}(r)$ is a nonnegative and nondecreasing function on $[0, \infty)$ such that $Q_{0}(0)=q^{*}$.

For $n=1$ the system (10)-(13) describes the situation when the moving ions are confined to the layer between two infinite planes in $\mathbb{R}^{3}, \pi_{1}=$ $\{(x, y, z): x=0\}$ and $\pi_{2}=\{(x, y, z): x=1\}$. The plane $\pi_{1}$ is charged with charge density $q^{*}$ and the plane $\pi_{2}$ is grounded.

An electrolyte contained in the infinite cylinder $\left\{(x, y, z): x^{2}+y^{2} \leq 1\right\}$, with fixed charge density $q^{*}$ on the $z$-axis, is described by (10)-(13) with $n=2$.

The case $n=3$ corresponds to the physical model of an electrolyte contained in the unit ball in $\mathbb{R}^{3}$ with a fixed charge $q^{*}$ at the origin.

We are interested in the existence, uniqueness and asymptotic behavior as $t \rightarrow \infty$ of the solution of the problem (10)-(13).

2. Stationary solutions. For $n=1$ and $n=2$ the stationary solutions to (10)-(12) can be expressed in analytic form. In dimension $n=3$ such a form of stationary solutions is not known and probably the equation is not integrable. In this case we resort to the phase plane method to prove the existence and uniqueness of solutions. The same method, though unnecessary, will also be used in much simpler one- and two-dimensional cases. Moreover, the phase plane method allows us to find a very simple proof of existence and uniqueness when analytic arguments need some tedious calculations.

One-dimensional case. In the one-dimensional case the stationary solutions of (10)-(12) satisfy

$$
\begin{gathered}
Q_{r r}-Q Q_{r}=0, \\
Q(0)=q^{*}, \quad Q(1)=\bar{q} .
\end{gathered}
$$

The equation (14) is equivalent to

$$
x^{\prime}=y, \quad y^{\prime}=x y, \quad '=d / d r,
$$

with $x=Q, y=Q_{r}$.

Figure 1 shows the phase portrait of (16).

For a given $q^{*}, \bar{q}$, we are looking for a solution $(x(r), y(r))$ to (16) satisfying $x(0)=q^{*}$ and $x(1)=\bar{q}$.

Let $(x(r ; \widetilde{y}), y(r ; \widetilde{y}))$ be a solution to $(16)$ such that $x(0, \widetilde{y})=q^{*}$ and $y(0, \widetilde{y})=\widetilde{y}$. It is easy to observe that the function $r(\widetilde{y})$ defined by $x(r(\widetilde{y}) ; \widetilde{y})$ 


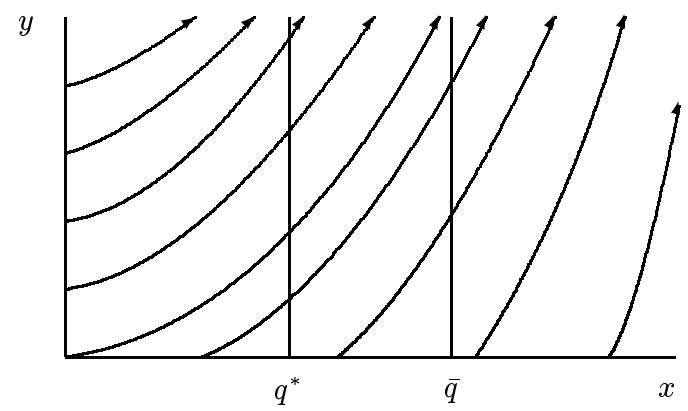

Fig. 1

$=\bar{q}$ is strictly decreasing and $r(\widetilde{y}) \rightarrow 0$ as $\widetilde{y} \rightarrow \infty$. The $x$-axis contains the singular points, hence $r(\widetilde{y}) \rightarrow \infty$ as $\widetilde{y} \rightarrow 0$. This implies the existence of a unique $\bar{y}$ such that $r(\bar{y})=1$. It means that $Q(r)=x(r ; \bar{y})$ is the unique solution to (14), (15).

Analytically we can solve the problem (14), (15) in the following way.

The equation (14) has the first integral

$$
Q_{r}-\frac{1}{2} Q^{2}=C .
$$

Integrating (17) we get an explicit form of the solution to (14) satisfying the initial data $Q(0)=q^{*}$ :

$$
\begin{gathered}
Q_{1}(r)=\frac{\sqrt{2 C} \tan (\sqrt{C / 2} r)+q^{*}}{1-\frac{q^{*}}{\sqrt{2 C}} \tan (\sqrt{C / 2} r)} \quad \text { if } C>0, \\
Q_{2}(r)=\frac{2 q^{*}}{2-q^{*} r} \quad \text { if } C=0, \\
Q_{3}(r)=\sqrt{-2 C} \frac{\left(q^{*}+\sqrt{-2 C}\right) \exp (-\sqrt{-2 C} r)-\left(\sqrt{-2 C}-q^{*}\right)}{\left(q^{*}+\sqrt{-2 C}\right) \exp (-\sqrt{-2 C} r)+\left(\sqrt{-2 C}-q^{*}\right)} \\
\text { if } C<0 .
\end{gathered}
$$

It follows from (19) that $Q_{2}(\bar{r})=\bar{q}$ if $\bar{r}=2 q_{0} /\left(\bar{q} q^{*}\right)$. Hence (see Fig. 1) the solution to the problem (14)-(15) has the form (19) if $2 q_{0}=\bar{q} q^{*}$, and (18) (resp. (20)) if $2 q_{0}>\bar{q} q^{*}$ (resp. $2 q_{0}<\bar{q} q^{*}$ ).

Two-dimensional case. For $n=2$ the stationary solutions to the problem (10)-(12) satisfy

$$
Q_{r r}-r^{-1} Q_{r}-(2 \pi)^{-1} Q Q_{r}=0
$$

with boundary conditions

$$
Q(0)=q^{*}, \quad Q(1)=\bar{q} .
$$


To analyze the problem of existence and uniqueness of solutions to (21), (22) we can use the phase plane method.

Introducing the functions $v(r)=(2 \pi)^{-1} r Q_{r}(r)$ and $w(r)=(2 \pi)^{-1} Q(r)$ we check that $v$ and $w$ satisfy the system of equations

$$
w^{\prime}=v, \quad v^{\prime}=v(2+w),
$$

where $^{\prime}=d / d s, s=\log r$. Figure 2 shows the phase portrait of (23).

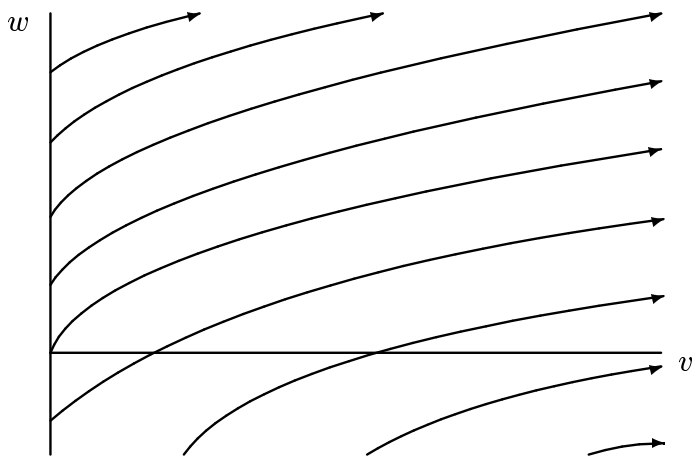

Fig. 2

The boundary data (22) takes the form

$$
w(-\infty)=q^{*}, \quad w(0)=\bar{q} .
$$

Analyzing Figure 2 we see that the problem (23), (24) has a unique solution.

Using the existence of the first integral $2 r Q_{r}-4 Q-\frac{1}{2 \pi} Q^{2}=C$ of the equation (21) we obtain the solution in the following form:

$$
\begin{aligned}
Q(r)= & \left(q^{*}\left(8 \pi+q^{*}+\bar{q}\right)+q_{0}\left(8 \pi+q^{*}\right) r^{\left(1+q^{*} /(4 \pi)\right) / 2}\right) \\
& \times\left(8 \pi+q^{*}+\bar{q}-q_{0} r^{\left(1+q^{*} /(4 \pi)\right) / 2}\right)^{-1} .
\end{aligned}
$$

It follows from formulas (18)-(20) and (25) that there are no solutions to the problem (10), (11) existing for all $t \geq 0$.

Three-dimensional case. For $n=3$, introducing the new variables

$$
v(r)=(4 \pi)^{-1} Q_{r}, \quad w(r)=(4 \pi)^{-1} Q / r, \quad s=\log r,
$$

we transform the problem of existence of stationary solutions to (10), (11) into the problem

$$
\begin{gathered}
w^{\prime}=v-w, \quad v^{\prime}=v(2+w), \quad '=d / d s, \\
w(0)=(4 \pi)^{-1} \bar{q}, \quad \lim _{s \rightarrow-\infty} w(s) e^{s}=(4 \pi)^{-1} q^{*} .
\end{gathered}
$$

Figure 3 shows the phase portrait of (27).

The origin is a saddle, $\gamma$ is its unstable manifold. Let $(w(s), v(s))$ be the solution to $(27)$ with initial data $w(0)=(4 \pi)^{-1} \bar{q}, v(0)=v \in[0, \bar{v})$ (see Fig. 3). 


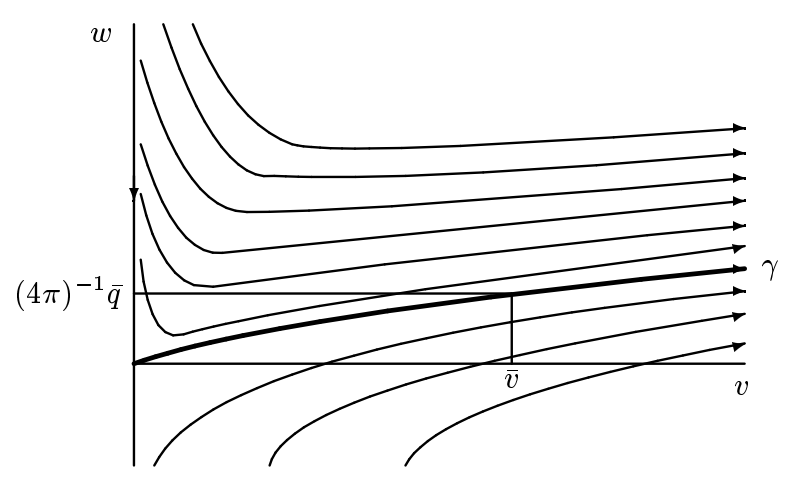

Fig. 3

This solution is defined on some maximal interval $(\alpha, \beta)$. It follows from the form of the equation (27) that $\alpha=-\infty$. The function $e^{s} w(s)$ is increasing (since its derivative is $e^{s} v(s)$ ), hence $\lim _{s \rightarrow-\infty} e^{s} w(s)=: L(v)$ exists. Obviously $L(0)=\bar{q}$ and $L(\bar{v})=0$, so there exists $v^{*} \in[0, \bar{v})$ such that $L\left(v^{*}\right)=q^{*}$. The uniqueness of $v^{*}$ follows from the monotonicity of $L(v)$.

The nonexistence of a solution to (10), (11) on $[0, \infty)$ for $n=3$ follows from the fact that the solution $(w(s), v(s))$ is contained between the lines $v=0$ and $w=v / 2$. Hence $v^{\prime} \geq 2 v+v^{2} / 2$, so the maximal interval of the existence of $(v(s), w(s))$ is $(-\infty, \beta), \beta<\infty$.

3. Nonstationary problem: One-dimensional case. For $n=1$ the equation (10) takes the form

$$
Q_{t}=Q_{r r}-Q Q_{r}
$$

which is called the Burgers equation. Using the Cole-Hopf transformation $Q=-2 v_{r} / v$ we can transform (29) into the heat equation ([6])

$$
v_{t}=v_{r r}
$$

with boundary data

$$
v_{r}(0, t)+\frac{q^{*}}{2} v(0, t)=0, \quad v_{r}(1, t)+\frac{\bar{q}}{2} v(1, t)=0 .
$$

The initial condition reads

$$
v(r, 0)=\exp \left(-\frac{1}{2} \int_{0}^{r} Q_{0}(s) d s\right) .
$$

Using the strong maximum principle and the Hopf lemma it is easy to prove that the solution $v$ to (30)-(32) is positive, $v_{r} \leq 0$ and $Q_{r} \geq 0$ (see [6]).

The standard Fourier method applied to the heat problem allows us to represent the solution $Q=-2 v_{r} / v$ in the following form. 
For $2 q_{0}>q^{*} \bar{q}$,

$$
Q(r, t)=-2 \frac{\sum_{k=0}^{\infty} a_{k} e^{-\lambda_{k}^{2} t} \lambda_{k}\left(\sin \left(\lambda_{k} r\right)+\frac{q^{*}}{2 \lambda_{k}} \cos \left(\lambda_{k} r\right)\right)}{\sum_{k=0}^{\infty} a_{k} e^{-\lambda_{k}^{2} t}\left(\frac{q^{*}}{2 \lambda_{k}} \sin \left(\lambda_{k} r\right)-\cos \left(\lambda_{k} r\right)\right)},
$$

where $\lambda_{k}>0, k=1,2, \ldots$, satisfies

$$
\frac{q_{0}}{2} \lambda_{k} \cot \lambda_{k}=\lambda_{k}^{2}+\frac{q^{*} \bar{q}}{4} .
$$

For $2 q_{0}=q^{*} \bar{q}$,

$$
Q(r, t)=-2 \frac{a_{0}+\sum_{k=1}^{\infty} a_{k} e^{-\lambda_{k}^{2} t} \lambda_{k}\left(\sin \left(\lambda_{k} r\right)+\frac{q^{*}}{2 \lambda_{k}} \cos \left(\lambda_{k} r\right)\right)}{a_{0}\left(r-\frac{2}{q^{*}}\right)+\sum_{k=1}^{\infty} a_{k} e^{-\lambda_{k}^{2} t}\left(\frac{q^{*}}{2 \lambda_{k}} \sin \left(\lambda_{k} r\right)-\cos \left(\lambda_{k} r\right)\right)}
$$

where $\lambda_{k}>\sqrt{\pi}, k=1,2, \ldots$, satisfies (34).

Finally, for $2 q_{0}<q^{*} \bar{q}$,

$$
Q(r, t)=\frac{A(r, t)}{B(r, t)}
$$

where

$$
\begin{aligned}
A(r, t)= & -2\left(a_{0} e^{-\lambda_{0}^{2} t} \lambda_{0}\left(e^{\lambda_{0} r}-\frac{2 \lambda_{0}+q^{*}}{2 \lambda_{0}-q^{*}} e^{-\lambda_{0} r}\right)\right. \\
& \left.+\sum_{k=1}^{\infty} a_{k} e^{-\lambda_{k}^{2} t} \lambda_{k}\left(\sin \left(\lambda_{k} r\right)+\frac{q^{*}}{2 \lambda_{k}} \cos \left(\lambda_{k} r\right)\right)\right), \\
B(r, t)= & a_{0} e^{-\lambda_{0}^{2} t}\left(e^{\lambda_{0} r}+\frac{2 \lambda_{0}+q^{*}}{2 \lambda_{0}-q^{*}} e^{-\lambda_{0} r}\right) \\
& +\sum_{k=1}^{\infty} a_{k} e^{-\lambda_{k}^{2} t}\left(\frac{q^{*}}{2 \lambda_{k}} \sin \left(\lambda_{k} r\right)-\cos \left(\lambda_{k} r\right)\right),
\end{aligned}
$$

$\lambda_{0}>0$ is the unique solution to

$$
e^{2 \lambda_{0}} \frac{\left(q^{*}-2 \lambda_{0}\right)\left(\bar{q}+2 \lambda_{0}\right)}{\left(q^{*}+2 \lambda_{0}\right)\left(\bar{q}-2 \lambda_{0}\right)}=1,
$$

and $\lambda_{k}, k=1,2, \ldots$, satisfy $(34)$.

It is easy to see that the solutions (33), (35), (36) tend to stationary ones as $t \rightarrow \infty$.

The Cole-Hopf substitution reduces the problem (28), (11), (13) on $[0, \infty)$ to a problem for the heat equation (for details see [8]):

$$
\begin{aligned}
& v_{t}=v_{r r}, \\
& \frac{q^{*}}{2} v(0, t)+v_{r}(0, t)=0, \\
& v(r, 0)=\exp \left(-\frac{1}{2} \int_{0}^{r} Q_{0}(s) d s\right)=: f(r) .
\end{aligned}
$$


The solution to (38) has the form ([5])

$$
v(r, t)=\int_{0}^{\infty} N_{1}(r, \xi, t) f(\xi) d x-2 \int_{0}^{t} K(r, t-\tau) g(\tau) d \tau,
$$

where $g$ is the unique solution to the integral equation

$$
g(t)=-\frac{q^{*}}{2} \int_{0}^{\infty} N_{1}(0, \xi, t) f(\xi) d \xi+q^{*} \int_{0}^{t} K(0, t-\tau) g(\tau) d \tau .
$$

Here

$$
\begin{aligned}
K(x, t) & =\frac{1}{\sqrt{4 \pi t}} \exp \left(-x^{2} /(4 t)\right), \\
N_{1}(x, \xi, t) & =K(x-\xi, t)+K(x+\xi, t), \\
N_{2}(x, \xi, t) & =K(x-\xi, t)-K(x+\xi, t) .
\end{aligned}
$$

For $q^{*}=0$ we can write the solution $Q$ and its derivative $Q_{r}$ in the explicit form

$$
\begin{gathered}
Q(r, t)=\frac{\left.\int_{0}^{\infty} N_{2}(r, \xi, t)\right) Q_{0}(\xi) f(\xi) d \xi}{\left.\int_{0}^{\infty} N_{1}(r, \xi, t)\right) f(\xi) d \xi}, \\
Q_{r}(r, t) \\
=\left(\int_{0}^{\infty} N_{1}(r, \xi, t)\left(Q_{0} f\right)^{\prime}(r, \xi, t) d \xi \int_{0}^{\infty} N_{1}(r, \xi, t) f(\xi) d \xi\right. \\
\left.+\left(\int_{0}^{\infty} N_{2}(r, \xi, t)\left(Q_{0} f\right)(r, \xi, t) d \xi\right)^{2} / 2\right)\left(\int_{0}^{\infty} N_{1}(r, \xi, t) f(\xi) d \xi\right)^{-2} .
\end{gathered}
$$

According to the physical interpretation $Q(r, t)$ should be a nondecreasing function of $r$, i.e. $Q_{r} \geq 0$. It is rather difficult to prove this property of $Q$ using $v$ (cf. (38)). However, we can prove that $Q_{0}^{\prime}>0$ implies $Q_{r}>0$ proceeding exactly as in [8, Th. 3]. The main tool in this proof is the Hopf lemma.

Now we are interested in the asymptotic behavior of solutions to (28), (11), (13) as $t \rightarrow \infty$. It follows from (41) that for $q^{*}=0$ the solution of the problem tends to 0 uniformly on each interval $[0, R]$ as $t \rightarrow \infty$. Using this fact we prove that for $q^{*}>0$ and bounded $Q_{0}^{\prime}(r)$ the solution $Q(r, t)$ to (28), (11), (13) tends to $q^{*}$ as $t \rightarrow \infty$.

Let $\widetilde{Q}$ be a solution of $(28)$ such that $\widetilde{Q}(0, t)=0$ and $\widetilde{Q}(r, 0)=Q_{0}(r)-q^{*}$. We claim that $\widetilde{Q}+q^{*}=: \bar{Q} \geq Q$. It is easy to verify that $W:=\bar{Q}-Q$ satisfies

$$
\begin{aligned}
& W_{t}=W_{r r}-\bar{Q} W_{r}-\bar{Q}_{r} W+q^{*} \bar{Q}_{r}, \\
& W(0, t)=0, \quad W_{0}(r, 0)=0 .
\end{aligned}
$$


It follows from (42) that $\bar{Q}_{r}$ is bounded, obviously $W$ is also bounded, hence we can apply the Phragmén-Lindelöf principle [9] which implies that $W \geq 0$. Hence $Q$ tends to $q^{*}$ as $t \rightarrow \infty$.

4. Nonstationary problem: Two- and three-dimensional case. Introducing the new variable $y=r^{n}$ we transform the problem (10)-(13) into

$$
\begin{aligned}
Q_{t} & =n^{2} y^{2-2 / n} Q_{y y}-n \sigma_{n}^{-1} Q Q_{y}, \\
Q(0, t) & =q^{*}, \\
Q(1, t) & =\bar{q}, \\
Q(y, 0) & =Q_{0}(y) .
\end{aligned}
$$

We assume that $Q_{0}(y) \geq 0$ is a nondecreasing function such that $Q_{0}(0)$ $=q^{*}, Q_{0}(1)=\bar{q}$ and $\bar{q} y^{k} \leq Q_{0}(y) \leq \widehat{q} y+q^{*}$ for some $k>0$ and $\widehat{q}>0$.

The essential difficulty is that the problem (44)-(47) is not uniformly parabolic (the diffusion coefficient is $y^{2-2 / n}$ ). Hence we cannot apply directly standard existence results. To overcome this difficulty we consider the approximation of (44)-(47) by uniformly parabolic problems

$$
Q_{t}=n^{2}(y+\varepsilon)^{2-2 / n} Q_{y y}-n \sigma_{n}^{-1} Q Q_{y}, \quad \varepsilon>0,
$$

with boundary and initial data (45)-(47).

It follows from the standard theory of uniformly parabolic problems (see [7, Ch. VI, Th. 5.2]) that for $Q_{0} \in C^{\alpha}([0,1])$, the problem $(45)-(48)$ has a unique solution $Q_{\varepsilon} \in C^{2+\alpha, 1+\alpha / 2}([0,1] \times[0, T])$ for each $T \geq 0$. Moreover, the estimate of the Hölder norm $\left\|Q_{\varepsilon}\right\|_{C^{\alpha, \alpha / 2}([\delta, 1] \times[0, T])} \leq C(\delta)$ holds for each $\delta>0$ with $C(\delta)$ independent of $\varepsilon$. This estimate allows us to choose a convergent subsequence $\left\{Q_{\varepsilon_{k}}\right\}$ which tends on $[\delta, 1] \times[0, T]$ to a function $Q$. From standard results ([7, Ch. III, Th. 10.1]) it follows that the family $\left\{Q_{\varepsilon}\right\}$ is bounded in $C^{m+\alpha,(m+\alpha) / 2}([y / 2,1] \times[t, T])$ for all integer $m$ and $\alpha \in(0,1)$. Obviously $Q(1, t)=\bar{q}$, and we must show that $Q(0, t)=q^{*}$.

We prove that

$$
Q(y, t) \leq \widehat{q} y+q^{*}=: \bar{Q}(y) .
$$

In fact,

$$
n^{2}(y+\varepsilon)^{2-2 / n} \bar{Q}_{y y}-n \sigma_{n}^{-1} \bar{Q} \bar{Q}_{y}-\bar{Q}_{t} \leq 0,
$$

$\bar{Q}(0)=q^{*}$ and $Q_{0}(y) \leq \widehat{q} y+q^{*}$. The comparison principle for the problem (45)-(48) holds, so if $Q^{\varepsilon}$ is a solution to (45)-(48), then $Q^{\varepsilon}(y, t) \leq \widehat{q} y+q^{*}$ for all $\varepsilon>0$. This implies (49).

In a similar way we can prove that $\underline{Q}(y):=\bar{q} y^{k} \leq Q(y, t)$. This inequality implies the estimate

$$
Q_{y}(1, t) \leq k \bar{q},
$$


which we will use in the proof of the boundedness of the derivative $Q_{y}(y, t)$ $=: U(y, t)$.

It is easy to prove that $U$ satisfies

$$
n^{2} y^{2-2 / n} U_{y y}+n^{2}(2-2 / n) U_{y}-n \sigma_{n}^{-1} U^{2}-n \sigma_{n}^{-1} Q U_{y}-U_{t}=0,
$$

$U(y, 0)=\left(Q_{0}\right)_{y}(y), U(0, t) \leq \widehat{q}, U(1, t) \leq k \bar{q}$.

The function $\bar{U}(y)=\bar{U}=\max \left(\widehat{q}, k \bar{q}, \max \left(Q_{0}\right)_{y}(y)\right)$ majorizes $U$. This can be proved exactly in the same way as the existence of an upper solution to $(44)-(47)$.

5. Asymptotic behavior of the solution to $(44)-(47)$. We prove that the solution $Q(y, t)$ to $(44)-(47)$ constructed above tends asymptotically, as $t$ goes to $\infty$, to a stationary solution $Q^{\mathrm{s}}(y)$ given at the end of Section 2. The key point in the proof is the existence of a Lyapunov function $W$ of the form

$$
\begin{aligned}
W(t)= & \int_{0}^{1}\left(Q_{y}\left(\log \left(Q_{y} / Q_{y}^{\mathrm{s}}\right)-1\right)+Q_{y}^{\mathrm{s}}\right) d y \\
& +\frac{1}{4 n \sigma_{n}} \int_{0}^{1} y^{2 / n-2}\left(Q-Q^{\mathrm{s}}\right)^{2} d y, \quad n=2,3 .
\end{aligned}
$$

Note that the estimate (49) implies that $\left(Q-Q^{\mathrm{s}}\right)^{2} \leq C y^{2}$ for some $C>0$. Hence the second integral in (51) makes sense and $W$ is well defined. It follows from the inequality $a(\log (a / b)-1)+b \geq 0$ valid for $a, b>0$ that the first integral in (51) is positive, hence $W \geq 0$. To prove that $W$ is the Lyapunov function we have to show that $W^{\prime}(t) \leq 0$. In fact,

$$
W^{\prime}(t)=\int_{0}^{1}\left(Q_{y}\right)_{t} \log \left(Q_{y} / Q_{y}^{\mathrm{s}}\right) d y+\frac{1}{2 n \sigma_{n}} \int_{0}^{1} y^{2 / n-2} Q_{t}\left(Q-Q^{\mathrm{s}}\right) d y .
$$

Integrating by parts we get

$$
\begin{aligned}
W^{\prime}(t)= & -\int_{0}^{1}\left(Q_{t} Q_{y y} / Q_{y}\right) d y+\int_{0}^{1}\left(Q_{t} Q_{y y}^{\mathrm{s}} / Q_{y}^{\mathrm{s}}\right) d y \\
& +\frac{1}{2 n \sigma_{n}} \int_{0}^{1} y^{2 / n-2} Q_{t} Q d y-\frac{1}{2 n \sigma_{n}} \int_{0}^{1} y^{2 / n-2} Q_{t} Q^{\mathrm{s}} d y \\
= & -\int_{0}^{1}\left(y^{2 / n-2} Q_{t}^{2} / Q_{y}\right) d y \leq-(\bar{U})^{-1} \int_{0}^{1} Q_{t}^{2} d y \leq 0,
\end{aligned}
$$

where $\bar{U}$ is given at the end of the previous section. Obviously $W^{\prime}(t)=0$ if and only if $Q=Q^{\mathrm{s}}$. 
Let $Q$ be a solution to (44)-(47). It follows from (52) and nonnegativity of $W$ that there exists a sequence $\left\{t_{n}\right\}$ such that $t_{n} \rightarrow \infty$ and $W^{\prime}\left(t_{n}\right) \rightarrow 0$ as $n \rightarrow \infty$. This implies that for every $0 \leq y \leq 1$,

$$
A\left(y, t_{n}\right):=\int_{0}^{y} Q_{t}\left(s, t_{n}\right) d s=\int_{0}^{y}\left(n^{2} y^{2-2 / n} Q_{y y}-n \sigma_{n}^{-1} /\left(2\left(Q^{2}\right)_{y}\right)\right) d s
$$

tends to 0 as $t_{n} \rightarrow \infty$. Integrating by parts we get

$$
\begin{aligned}
A(y, t)= & n^{2} y^{2-2 / n} Q_{y}+\int_{0}^{y} n^{2}\left(2-\frac{2}{n}\right)\left(1-\frac{2}{n}\right) s^{-2 / n} Q d s \\
& -n^{2}\left(2-\frac{2}{n}\right) y^{1-2 / n} Q-n \sigma_{n}^{-1} Q^{2} / 2+n \sigma_{n}^{-1}\left(q^{*}\right)^{2} / 2 .
\end{aligned}
$$

The estimate (50) implies that the family $Q\left(\cdot, t_{n}\right)$ is compact in $C^{0}$ topology. Hence we may assume that $Q\left(\cdot, t_{n}\right) \rightarrow \bar{Q}(\cdot)$ uniformly on $[0,1]$. Because $A\left(y, t_{n}\right) \rightarrow 0$ we conclude that $Q_{y}\left(\cdot, t_{n}\right)$ converges almost uniformly on $(0,1]$ to $\bar{Q}_{y}$, and $\bar{Q}$ satisfies

$$
\begin{aligned}
n^{2} y^{2-2 / n} \bar{Q}_{y} & +\int_{0}^{y} n^{2}\left(2-\frac{2}{n}\right)\left(1-\frac{2}{n}\right) y^{-2 / n} \bar{Q} \\
& -n^{2}\left(2-\frac{2}{n}\right) y^{1-2 / n} \bar{Q}-n \sigma_{n}^{-1} \bar{Q}^{2}+n \sigma_{n}^{-1}\left(q^{*}\right)^{2} / 2=0 .
\end{aligned}
$$

From (53) we see that the function $\bar{Q}$ is in $C^{2}(0,1)$. Differentiating (53) with respect to $y$ we see that $n^{2} y^{2-2 / n} \bar{Q}_{y y}-n \sigma_{n}^{-1} \bar{Q} \bar{Q}_{y}=0$. Hence $\bar{Q}$ is a stationary solution to the problem (44)-(47). In this way we proved that $Q\left(\cdot, t_{n}\right) \rightarrow Q^{\mathrm{s}}(\cdot)$ in $C^{1}$-topology almost uniformly on $(0,1]$. This implies that $W\left(t_{n}\right) \rightarrow 0$, so $\int_{0}^{1}\left(Q(y, t)-Q^{\mathrm{s}}(y)\right)^{2} d y \rightarrow 0$ as $t \rightarrow \infty$. The convergence of $Q(\cdot, t)$ to $Q^{\mathrm{s}}$ in $L^{1}$ and boundedness of $Q_{y}$ imply the convergence of $Q$ to $Q^{\mathrm{s}}$ uniformly on $[0,1]$.

Acknowledgments. The authors would like to thank P. Biler, G. Karch and A. Krzywicki for numerous discussions during the preparation of this paper.

\section{References}

[1] P. Biler, Existence and asymptotics of solutions for a parabolic-elliptic system with nonlinear no-flux boundary conditions, Nonlinear Anal. 19 (1992), 1121-1136.

[2] P. Biler, W. Hebisch and T. Nadzieja, The Debye system: existence and long time behavior of solutions, ibid. 23 (1994), 1189-1209.

[3] P. Biler and T. Nadzieja, A singular problem in electrolytes theory, Math. Methods Appl. Sci. 20 (1997), 767-782. 
[4] P. Biler and T. Nadzieja, Nonlocal parabolic problems in statistical mechanics, Proc. Second World Congress of Nonlinear Analysts, Nonlinear Anal. 30 (1997), $5343-5350$

[5] J. R. Cannon, The One-Dimensional Heat Equation, Addison-Wesley, New York, 1984.

[6] A. Krzywicki and T. Nadzieja, A nonstationary problem in the theory of electrolytes, Quart. Appl. Math. 50 (1992), 105-107.

[7] O. A. Ladyženskaja, V. A. Solonnikov and N. N. Ural'ceva, Linear and Quasilinear Equations of Parabolic Type, Amer. Math. Soc. , Providence, R.I., 1988.

[8] T. Nadzieja, A model of radially symmetric cloud of self-attracting particles, Appl. Math. (Warsaw) 23 (1995), 169-178.

[9] M. H. Protter and H. F. Wein berger, Maximum Principles in Differential Equations, Springer, New York, 1984.

[10] I. Rubinstein, Electro-Diffusion of Ions, SIAM Stud. Appl. Math. 11, Philadelphia, 1990.

Tadeusz Nadzieja

Institute of Mathematics

Technical University of Zielona Góra

Podgórna 50

65-246 Zielona Góra, Poland

E-mail: T.Nadzieja@im.pz.zgora.pl
Andrzej Raczyński Mathematical Institute University of Wrocław Pl. Grunwaldzki 2/4 50-384 Wrocław, Poland E-mail: aracz@math.uni.wroc.pl 